\title{
Niveaux hormonaux plasmatiques de poules albinos (sal-c) et non albinos $(\mathrm{S})$
}

\author{
P Mérat ${ }^{1}$, E Decuypere ${ }^{2}, \mathrm{~J}$ Buyse $^{2}$ \\ ${ }^{1}$ Institut national de la recherche agronomique, laboratoire de génétique factorielle, \\ 78352 Jouy-en-Josas cedex, France; \\ ${ }^{2}$ Katholieke Universiteit Leuven, Laboratorium voor Fysiologie der Huisdieren, Heverlee, \\ Belgique
}

(Reçu le 2 novembre 1993; accepté le 3 février 1994)

Résumé - Des poules albinos (mutation $\mathrm{s}^{\mathrm{al}-\mathrm{c}}$ ) et non-albinos (colorées) de même origine ont été comparées pour des niveaux plasmatiques hormonaux, triiodothyronine (T3), thyroxine (T4), hormone de croissance (GH) et corticostérone, avant l'entrée en ponte puis après 3 à 4 mois de ponte. Le rapport T3/T4 était plus élevé chez les poules albinos que chez les non-albinos aux 2 âges; cette différence s'approche de la signification après l'entrée en ponte. La variance intra-génotype de ce rapport est plus élevée pour les poules $\mathrm{s}^{\mathrm{al}-\mathrm{c}}$ que pour les non-albinos $(P<0,01)$. D'autre part, le taux de l'hormone de croissance ne diffère pas entre génotypes avant l'entrée en ponte, mais en période de ponte il est significativement plus élevé chez les poules $\mathrm{s}^{\text {al }}$. Il existe donc certaines différences dans l'équilibre hormonal des 2 génotypes. On peut espérer que ceci aide à l'interprétation des effets trouvés associés au gène albinos sur la production d'œufs.

poule / albinisme lié au sexe / hormone thyroïdienne / hormone de croissance / corticostérone

Summary - Plasma hormone levels of albino $\left(\mathrm{s}^{\mathrm{al}-\mathrm{c}}\right)$ and non-albino hens. Albino $\left(s^{\mathrm{al}-\mathrm{c}}\right)$ and non-albino (colored) hens from the same origin were compared for several plasmatic hormonal levels (triiodothyronine (T3), thyroxine (T4), growth hormone (GH), and corticosterone) before the onset of laying and after 3-4 months of production. The T3/T4 ratio was higher among albino than among non-albino females at the 2 ages; this difference approached significance after sexual maturity. The within-genotype variance of this ratio was higher for $s^{\text {al-c }}$ hens than for non-albino ones $(\mathrm{P}<0.01)$. On the other hand, the GH level did not differ between the 2 genotypes before the onset of laying, but during laying it was significantly higher for the $s^{\text {al-c }}$ females. There are therefore some differences in the general hormonal balance of the 2 genotypes. It is hoped that this may help in an interpretation of the effects on egg production associated with the albino gene.

domestic fowl / sex-linked albino / thyroid hormone / growth hormone / corticosterone 


\section{INTRODUCTION}

L'albinisme imparfait lié au sexe chez la poule $\left(\mathrm{s}^{\mathrm{al}}\right)$ comporte plusieurs origines mutationnelles connues (Silversides et Crawford, 1990). A partir de l'une de ces origines (obtenue puis reproduite au laboratoire de génétique factorielle, Jouy-enJosas, à partir de l'University of Connecticut, Storrs, États-Unis, et correspondant à la mutation $\mathrm{s}^{\text {al-c }}$, nous avons montré (Mérat et Bordas, 1989) que des poules albinos, comparées à des poules non albinos de même origine, étaient supérieures à ces dernières pour plusieurs critères de production d'œufs en cages individuelles, avec un éclairage artificiel d'intensité lumineuse élevée, mais non à faible intensité lumineuse. Ceci a été vérifié plusieurs fois depuis dans les mêmes conditions, en particulier par Bordas et Mérat (1992). La reproduction étant contrôlée par des hormones, ceci fait supposer l'existence de différences hormonales entre poules albinos et non albinos. Silversides et al (1992) ont comparé des poulets albinos et non albinos pour le niveau de la mélatonine et de 2 enzymes associées, hydroxyindole O-methyl transférase (HIOMT) et N-acétyl transférase (NAT) à diverses heures du cycle nycthéméral, dans le plasma, la rétine et l'épiphyse; d'autres poulets ont été comparés en lumière ou obscurité constante. La seule différence significative trouvée entre génotypes concernait l'enzyme HIOMT en lumière et en obscurité constantes. D'autre part Silversides et al (1993) ont étudié le taux de LH plasmatique avant l'entrée en ponte et en début de ponte. Ce taux était légèrement inférieur pour les poules $\mathrm{s}^{\text {al }}$ à $17 \mathrm{sem}$ puis avant $33 \mathrm{sem}$.

À notre connaissance, on ne sait rien sur d'autres niveaux hormonaux moins directement impliqués dans la ponte que les précédents, en relation avec l'albinisme imparfait lié au sexe. Il nous a paru intéressant, compte tenu des interactions entre de nombreux niveaux hormonaux, de déterminer le niveau des hormones thyroïdiennes, triiodothyronine (T3) et thyroxine (T4), de l'hormone de croissance $(\mathrm{GH})$ et de la corticostérone dans le plasma de poules albinos et non albinos de même origine, soumises aux conditions (en particulier d'intensité lumineuse) qui avaient auparavant favorisé le génotype albinos pour la production d'œufs.

\section{MATÉRIEL ET MÉTHODES}

Le gène $\mathrm{s}^{\mathrm{al}-\mathrm{c}}$ (désigné, par abréviation, par $\mathrm{s}^{\text {al }}$ dans ce qui suit) était maintenu en ségrégation dans une lignée du laboratoire de type «œuf brun» depuis 1979 (Mérat et Bordas, 1989). Les femelles non albinos argentées (S) et albinos $\left(\mathrm{s}^{\mathrm{al}}\right)$ utilisées dans le présent travail étaient issues du croisement de coqs hétérozygotes $\left(\mathrm{S} / \mathrm{s}^{\mathrm{a}}\right)$ et de poules s ${ }^{+}$de cette lignée. En février 1992, 39 poulettes argentées et 32 poulettes albinos, identifiées par la couleur de l'œil, étaient écloses et élevées au sol jusqu'à l'âge de $17 \mathrm{sem}$ dans une poussinière sans fenêtres. Elles recevaient $10 \mathrm{~h}$ de lumière par $24 \mathrm{~h}$, avec une intensité lumineuse comprise entre 0,5 et 2 lux à la hauteur des poussins. À $17 \mathrm{sem}, 25$ poulettes $\mathrm{s}^{\text {al }}$ et 23 poulettes $S$ étaient placées en cages individuelles sur 2 niveaux dans une cellule éclairée par des tubes fluorescents. Les poulettes recevaient $14 \mathrm{~h}$ d'éclairement par $24 \mathrm{~h}$ avec une intensité lumineuse moyenne respectivement de 560 et 246 lux en façade des cages des niveaux supérieur et inférieur. La température était maintenue constante à $22^{\circ} \mathrm{C} \pm 1{ }^{\circ} \mathrm{C}$. L'aliment, distribué ad libitum en farine, contenait $15,5 \%$ de protéines totales, $11,05 \mathrm{MJ} / \mathrm{kg}$ 
d'énergie métabolisante et $3,4 \%$ de calcium. Ces conditions étaient très proches de celles trouvées précédemment favorables au génotype $\mathrm{s}^{\text {al }}$ (Mérat et Bordas, 1989; Bordas et Mérat, 1992).

Les poulettes étaient pesées à $16 \mathrm{sem}$. L'âge au $1^{\mathrm{er}}$ œuf était relevé, ainsi que le nombre d'œufs jusqu'à l'âge de 34 sem et l'intensité de ponte (rapport du nombre d'œufs au nombre de jours de contrôle depuis l'entrée en ponte) pour la même période.

Un prélèvement sanguin (environ $1,5 \mathrm{cc}$ de sang total) était fait sur chaque poule le matin, avant l'entrée en ponte (entre les âges de 17 et $18 \mathrm{sem}$ ) puis entre les âges de 34 et 35 sem. Les plasmas congelés étaient envoyés au Laboratorium voor Fysiologie der Huisdieren, Université catholique de Louvain, Heverlee, Belgique, pour détermination des taux hormonaux.

L'hormone de croissance aviaire était mesurée par le dosage radio-immunologique homologue développé par Berghman et al (1988). Le coefficient de variation intraessai était de $4 \%$. Les mesures de concentration plasmatique de T3 étaient faites par dosage radio-immunologique utilisant un antisérum commercial vis-à-vis de T3 (Mallmckrodt) en combinaison avec un traceur spécifique (In 321, Amersham). Le coefficient de variation intra-essai était de 2,9\% (Huybrechts et al, 1989). Les concentrations plasmatiques de T4 étaient déterminées en utilisant un traceur (Amersham) et un antisérum de lapin anti-T4 réalisé au laboratoire. Cet antisérum présentait une réaction croisée de $0,16 \%$ avec T3 et un coefficient de variation intraessai de $3,2 \%$.

Les concentrations de corticostérone étaient mesurées par dosage radio-immunologique en employant un kit commercial (IDSltd).

Les niveaux plasmatiques de chaque hormone, ainsi que le rapport T3/T4, étaient d'abord comparés séparément à chaque âge par un test t. Puis, pour la moyenne de 2 âges calculée individuellement pour les poules pour lesquelles les 2 valeurs avaient pu être déterminées, une analyse de variance était faite (analyse par split plot de mesures répétées avec les facteurs contrôlés «génotype» et «âge» - SAS Institute, 1988). Les tests faits séparément pour chaque âge se justifiaient dans la mesure où l'on pouvait supposer que les taux hormonaux considérés avant maturité sexuelle et plusieurs mois après celle-ci avaient des significations biologiques éventuellement différentes et représentaient en fait des variables distinctes.

\section{RÉSULTATS ET DISCUSSION}

Le poids moyen à l'âge de 16 sem était voisin pour les poules albinos et nonalbinos : respectivement 1440 et $1445 \mathrm{~g}$. L'âge au $1^{\mathrm{er}}$ œuf des mêmes génotypes, respectivement $152,3 \mathrm{j}$ et $147,3 \mathrm{j}$ suggérait une précocité légèrement plus grande des poules non-albinos. Les nombres d'œufs jusqu'à 34 sem, respectivement 73,6 et 74,2 , et les intensités de ponte (respectivement 89,7 et 89,0 ) ne différaient pas significativement sur cet échantillon limité. Il en était de même de la mortalité depuis la mise en cages ( 2 poules colorées et 3 poules $\mathrm{s}^{\mathrm{al}}$ ).

Le tableau I indique les valeurs moyennes des niveaux hormonaux plasmatiques mesurés, pour chacun des 2 prélèvements sanguins (âges 17 et 34 sem), ainsi que pour la moyenne de ces 2 prélèvements pour chaque hormone, basée sur la valeur moyenne de chaque individu ayant été mesuré aux 2 dates. 
Tableau I. Niveaux hormonaux avant et après entrée en ponte pour des poules albinos $\left(\mathrm{s}^{\mathrm{al}-\mathrm{c}}\right)$ et non-albinos $(\mathrm{S})$.

\begin{tabular}{|c|c|c|c|c|}
\hline \multirow{2}{*}{\multicolumn{2}{|c|}{$\begin{array}{c}\text { Variable }^{a} \text { et } \hat{a} g e \\
(A=17 \mathrm{~s}, B=34 \mathrm{~s})\end{array}$}} & \multicolumn{2}{|c|}{ Valeur moyenne } & \multirow[t]{2}{*}{ Signification } \\
\hline & & $s^{a l-c}$ & $S$ & \\
\hline \multirow[t]{3}{*}{$\mathrm{T} 3(\mathrm{ng} / \mathrm{ml})$} & $\mathrm{A}$ & 0,88 & 0,72 & \multirow{3}{*}{$\begin{array}{c}\text { NS } \\
\mathrm{NS} \\
b\end{array}$} \\
\hline & $\mathrm{B}$ & 0,73 & 0,70 & \\
\hline & moyenne & 0,79 & 0,71 & \\
\hline \multirow[t]{3}{*}{$\mathrm{T} 4(\mathrm{ng} / \mathrm{ml})$} & A & 11,83 & 11,93 & \multirow{3}{*}{$\begin{array}{c}\mathrm{NS} \\
\mathrm{NS} \\
b\end{array}$} \\
\hline & $\mathrm{B}$ & 6,96 & 7,86 & \\
\hline & moyenne & 9,46 & 9,90 & \\
\hline \multirow[t]{3}{*}{$10 \mathrm{~T} 3 / \mathrm{T} 4$} & $\mathrm{~A}$ & 0,73 & 0,61 & \multirow{3}{*}{$\mathrm{P}<\underset{b}{\mathrm{NS}} 0,10$} \\
\hline & $\mathrm{B}$ & 1,17 & 0,97 & \\
\hline & moyenne & 0,96 & 0,78 & \\
\hline \multirow[t]{3}{*}{$\mathrm{GH}(\mathrm{ng} / \mathrm{ml})$} & $\mathrm{A}$ & 4,91 & 5,00 & \multirow{3}{*}{$\begin{array}{c}\mathrm{NS} \\
\mathrm{P}<\underset{b}{0,02}\end{array}$} \\
\hline & B & 5,87 & 3,78 & \\
\hline & moyenne & 5,39 & 4,38 & \\
\hline \multirow[t]{3}{*}{ Corticostérone $(\mathrm{ng} / \mathrm{ml})$} & A & 1,05 & 1,21 & NS \\
\hline & $\mathrm{B}$ & 1,29 & 1,45 & NS \\
\hline & moyenne & 1,18 & 1,32 & $b$ \\
\hline
\end{tabular}

${ }^{a}$ Les effectifs sont compris entre 23 et 20 par génotype selon la variable et la période, sauf pour GH en période B (respectivement $19 \mathrm{~S}$ et $16 \mathrm{~s}^{\mathrm{al}-\mathrm{c}}$ pour lesquelles un dosage a pu être fait). ${ }^{b} c f$ tableau II. ${ }^{c}$ NS $=$ non significatif au seuil de $5 \%$.

Tableau II. Valeurs moyennes globales par âge et analyse de variance (effets génotype et âge) pour chaque niveau hormonal.

\begin{tabular}{|c|c|c|c|c|c|}
\hline \multirow{2}{*}{$\begin{array}{l}\text { Variable } \\
\text { T3 }\end{array}$} & \multicolumn{2}{|c|}{$\begin{array}{cc}\text { Valeurs moyennes par âge } \\
A\end{array}$} & \multicolumn{3}{|c|}{$\begin{array}{l}\text { Source de variation } \\
\text { génotype âge interaction }\end{array}$} \\
\hline & 0,80 & 0,72 & NS & NS & NS \\
\hline $\mathrm{T} 4$ & 11,88 & 7,41 & NS & $* * *$ & NS \\
\hline $10 \mathrm{~T} 3 / \mathrm{T} 4$ & 0,67 & 1,07 & NS & $* * *$ & NS \\
\hline GH & 4,96 & 4,83 & NS & NS & $*$ \\
\hline Corticostérone & 1,13 & 1,37 & NS & NS & NS \\
\hline
\end{tabular}

$a * p<0,05 ; * * * p<0,001$. NS : non significatif.

Le tableau II indique les valeurs moyennes par âge, et la signification des effets «âge» et «génotype» à partir de l'analyse de variance.

Les valeurs de T3 et T4 prises séparément ne diffèrent à aucun des 2 âges entre génotypes. Le rapport T3/T4 est toujours plus élevé chez les poules albinos, mais il ne diffère pas non plus significativement à chaque âge, ni pour la moyenne des 2 âges. Ce dernier point est confirmé par l'analyse de variance à 2 facteurs. Cependant, pour le second âge mesuré (après entrée en ponte), une supériorité des poules $\mathrm{s}^{\text {al }}$ 
pour ce rapport est suggérée $(P<0,10)$ malgré la variabilité élevée des valeurs individuelles. On peut ajouter que, à chaque âge ainsi que pour la moyenne des 2 âges, la variance intra-génotype du rapport T3/T4 est significativement plus élevée pour les poules $\mathrm{s}^{\mathrm{al}-\mathrm{c}}$ que pour les poules $\mathrm{S}$ (respectivement $\mathrm{F}=3,98, P<0,01$; $\mathrm{F}=2,30, P<0,05$ et $\mathrm{F}=3,19, P<0,01$ aux 2 âges et pour la moyenne des 2 âges). Il en est de même de la variance du taux de T3 avant l'entrée en ponte. Il y a donc une tendance à un étalement plus grand du rapport T3/T4 vers les valeurs élevées pour le génotype albinos, plutôt qu'une différence de la valeur moyenne seule. En tenant compte de l'hétérogénéité des variances, l'application du test indiqué par Snedecor et Cochran (1969) pour comparer les moyennes à chaque âge, au lieu du test $\mathrm{t}$ classique, ne change pas les premières conclusions sur l'absence de signification des différences entre moyennes au seuil 5\% pour les variables T3 et T4 et leur rapport.

Une autre différence significative est observée concernant l'hormone de croissance, non différente entre génotypes avant maturité sexuelle, mais significativement plus élevée chez lez poules $\mathrm{s}^{\mathrm{al}}$ après l'entrée en ponte. Ceci est reflété d'une autre façon par l'interaction significative entre âge et génotype observée pour cette hormone au tableau II.

Il est difficile d'interpréter pour l'instant ces différences, et en particulier de savoir si elles peuvent contribuer à expliquer la réponse différentielle des pondeuses albinos et non-albinos à l'intensité lumineuse (Mérat et Bordas, 1989), non observée, il est vrai, dans le présent échantillon, dont la taille réduite doit cependant être rappelée. Nous n'avons pas connaissance de données publiées quant à une corrélation éventuelle entre des critères de production d'œufs et le niveau des hormones T3, T4, GH et corticostérone, non plus qu'entre ce niveau et une réponse des pondeuses à l'intensité lumineuse. Seul a été suggéré parfois un effet de la photopériode sur les hormones thyroïdiennes, en particulier chez la caille japonaise (Peczely et al, 1979, 1980; Oishi et Konishi, 1978) et chez le dindon (Follett et Nicholls, 1984; Scanes et al, 1979; Lien et Siopes, 1989); on peut citer aussi une remarque de Sturkie (1976). Cependant une relation possible entre ces suggestions et nos observations n'est pas évidente pour l'instant. D'autre part, Bartha (1993) indique qu'il peut y avoir une relation entre une baisse de la production extrathyroïdienne de $\mathrm{T} 3$ et un nombre diminué de récepteurs à $\mathrm{GH}$ : on peut se demander si cette suggestion est à rapprocher des effets qui apparaissent ici associés à $\mathrm{s}^{\mathrm{al}}$, à la fois sur le taux de GH et sur le rapport T3/T4. Enfin, nous n'avons pas actuellement de mesures de métabolisme pouvant être mises en rapport avec le niveau de T3 et $\mathrm{T} 4$ chez les poules $\mathrm{s}^{\mathrm{al}}$ et $\mathrm{S}$.

Quoi qu'il en soit, tout progrès futur dans la compréhension des effets associés au gène $\mathrm{s}^{\mathrm{al}}$, en particulier sur la production d'œufs, peut se révéler utile pour une utilisation potentielle de ce gène dans la sélection avicole. 


\section{RÉFÉRENCES}

Bartha T (1993) Thyroid hormone metabolism in broiler chickens as influenced by exogenous and endogenous factors. Thèse, Katholieke Universiteit Leuven, Belgique Berghman L, Van Beeumen J, Decuypere E, Kühn ER, Vandesande F (1988) Onestep purification of chicken growth hormone from a crude pituitary extract by use of a monoclonal immunoadsorbant. J Endocrinol 118, 381-387

Bordas A, Mérat P (1992) Egg production response of sex-linked albino $\left(\mathrm{s}^{\mathrm{al}}\right)$ and colored (S) hens to high and low light intensities during brooding rearing. Genet Sel Evol 24, 171-177

Follett BK, Nicholls TJ (1984) Photorefractoriness in Japanese quail : possible involvement of the thyroid gland. J Exp Zool 232, 573-580

Huybrechts LM, Michielsen R, Darras V, Buonomo FC, Kühn ER, Decuypere E (1989) Effect of the sex-linked dwarf gene on thyrotropic and somatotropic axes in the chick embryo. Reprod Nutr Dev 29, 219-226

Lien RJ, Siopes TD (1989) Turkey plasma thyroid hormone and prolactin concentrations throughout an egg laying cycle and in relation to photorefractoriness. Poult Sci $68,1409-1417$

Mérat P, Bordas A (1989) Differential response of sex-linked albino $\left(\mathrm{s}^{\text {al }}\right)$ and silver (S) hens to high and low light intensity. Brit Poult Sci 30, 807-813

Oishi T, Konishi T (1978) Effects of photoperiod and temperature on testicular and thyroid activity of the Japanese quail. Gen Comp Endocrinol 36, 250-252

Peczely P, Pethes G, Rudas P (1980) Interrelationship between thyroid and gonadal function in female Japanese quail kept under short and long photoperiods. $J$ Endocrinol 87, 55-63

Peczely P, Astier H, Jallageas M (1979) Reciprocal interactions between testis and thyroid in the Japanese quail. Gen Comp Endocrinol 37, 400-404

SAS Institute (1988) Users guide: statistics. Cary, USA

Scanes CG, Shamp PJ, Harvey S, Godeden P, Chadwick A, Newcomer HS (1979) Variations in plasma prolactin, thyroid hormones, gonadal steroids and growth hormone in turkeys during the induction of egg laying and molt by different photoperiods. Brit Poult Sci 20, 143-148

Silversides FG, Crawford RD (1990) Genetic aspects of a new mutation to sex-linked imperfect albinism $\left(\mathrm{s}^{\mathrm{al}-\mathrm{s}}\right)$ in chickens. Genet Sel Evol 22, 447-455

Silversides FG, Voisin P, Ravault JP, Mérat P (1992) Effects of light on melatonin and two enzymes leading to its production in albino $\left(\mathrm{s}^{\mathrm{al-c}}\right)$ and non-albino chickens. Gen Comp Endocrinol 88, 328-333

Silversides FG, Williams JW, Mérat P (1993) Effects of sex-linked imperfect albinism in the chicken $\left(\mathrm{s}^{\mathrm{al}-\mathrm{c}}\right)$ on luteinizing hormone concentrations and early egg production. Brit Poult Sci 34, 1011-1019

Snedecor GW, Cohran WG (1969) Statistical Methods Applied to Experiments in Agriculture and Biology, Iowa State University Press, Ames, Iowa, 6e éd, 114-116 Sturkie PD (1976) Avian physiology. Springer, New York, 3e ed, $400 \mathrm{p}$ 